\title{
Bounds on the mixing of the down-type quarks with vector-like singlet quarks
}

\author{
L. Lavoura and João P. Silva \\ Department of Physics, Carnegie-Mellon University, \\ Pittsburgh, Pennsylvania 15213, U.S.A.
}

DOE/ER/45120--TI

DE93 002766

September 8, 1992

\begin{abstract}
We derive bounds on the mixing of the standard charge $-1 / 3$ quarks with vector-like isosinglet quarks, as they exist in some extensions of the standard model. We make no assumptions about the unitarity or any other features of the mixing matrix. We find that the mixing is quite constrained: we are able to set bounds on all the extra parameters which arise in the mixing matrix (CKM matrix), except on two phases. The assumption that there exists only one exotic quark leads to some extra relationships among the parameters of the mixing matrix.
\end{abstract}

\section{Introduction}

In the quest for physics beyond the Standard Model (SM), attention has been paid to the possible existence of fermions with non-canonical $S U(2) \times U(1)$ assignments $[1,2]$. Such "exotic" fermions arise in various extensions of the SM, and in particular they may be needed to cancel anomalies in theories with extended gauge groups. An example of this is the Grand Unified Theory based on the gauge group $E_{6}$. That theory, in its supersymmetric version, arises as the result of some compactifications of superstring models. In it, each family belongs to a representation 27 of $E_{f}$, which is anomalyfree; that representation contains the known fifteen fermions, and also a vector-like singlet quark of charge $-1 / 3$, a vector-like doublet of (charged and neutral) leptons, and two more neutrinos. In this paper we are concerned with the possible presence, in some extensions of the SM, of vector-like singlet quarks with charge $-1 / 3$. 'Taking a phenomenological point of view, we want to study the bounds that the present experimental results set on the mixing of those exotic quarks with the usual charge $-1 / 3$ (down-type) quarks.

In $E_{t}$, there is one exotic down-type quark in each generation, and therefore, assuming the existence of only three generations, there are three vector-like quarks. Their mixing with the three ordinary quarks of course involves a large number of parameters, 
and the complete mixing matrix is difficult to parametrize. Previous authors have usually done a number of simplifying assumptions in order to avoid this problem. In particular, in a recent study [3], Nardi, Roulet and Tommasini, based on the stringent experimental limits on Flavor-Changing Neutral Currents (FCNC), chose to simplify the problem by assuming these to be absent. They did this by assuming that the mixing matrix is such that each iso-singlet quark mixes with one and only one ordinary quark. We find this assumption rather unnatural. Other authors $[4,5]$ have assumed instead that only one exotic quark exists, instead of three. On the other hand, those authors allow for the presence of FCNC, and consider the experimental bounds on them. But one may question oneself whether the presence of more than one vector-like quark might not change the validity of some of their bounds, or whether a large number of un-constrained parameters would not then arise.

In this paper we make no such assumptions, and treat the problem in all its generality, assuming the presence of an arbitrary number of vector-like down-type quarks. We are able to do this because we notice that, whatever the number of exotic quarks may be, only a limited number of parameters arises in the mixing of the relevant sector of the down, strange and bottom quarks. Remarkably, we find that the present experimental information is already enough to set stringent bounds on most of the new parameters which arise in the mixing. Some of the original features of our work are: the use of a Wolfenstein-type parametrization for the mixing matrix, in order to manage in a simple way the different orders of magnitude of the various mixing parameters; and the consideration not only of the bounds on the existence of FCNC, but also of the ones coming from the analysis of the $Z$ decays.

The plan of this paper is as follows. In section 2 we write down the charged-and neutral-current couplings of the quarks in a model with isosinglet vector-like clown-type quarks, leaving the details of their derivation to an Appendix. In the third section we parametrize the mixing matrix of the known three down-type quarks, without making any assumptions about its unitarity, or the unitarity of any extension of it. In the fourth section we derive the experimental bounds on the various parameters. In the fifth section we show that the assumption of the existence of only one exotic quark leads to some relationships among the otherwise independent parameters. The conclusions are given in the sixth section. In the Appendix we work out at length the couplings of the quarks to the vector and scalar bosons in a $S U(2) \times U(1)$ model with an arbitrary number of iso-singlet, iso-doublet and mirror quarks.

\section{The mixing matrices}

We consider the three-generation standard $S U(2) \times U(1)$ model with the addition of $n_{\wedge}$. exotic quarks of electric charge $-1 / 3$, for which both chirality components are singlets of $\mathrm{SU}(2)$, and no other varieties of exotic fermions. The charged-current couplings of the quarks to the $W$ boson are, as usual, governed by the (generalized) Cabibbo- 


\section{DISCLAIMER}

This report was prepared as an account of work sponsored by an agency of the United States Government. Neither the United States Government nor any agency thereof, nor any of their employees, makes any warranty, express or implied, or assumes any legal liability or responsibility for the accuracy, completeness, or usefulness of any information, apparatus, product, or process disclosed, or represents that its use would no: infringe privately owned rights. Reference herein to any specific commercial product, process, or service by trade name, trademark, manufacturer, or otherwise does not necessarily constitute or imply its endorsement, recommendation, or favoring by the United States Government or any agency thereof. The views and opinions of authors expressed herein do not necessarily state or reflect those of the United States Government or any agency thereof. 
Kobayashi-Maskawa (CKM) matrix $V$. The charged-current Lagrangian reads

$$
\mathcal{L}_{\text {H }}=\frac{g}{\sqrt{2}} \bar{u}_{L} \gamma^{\mu} V d_{L} W_{\mu}^{+}+\text {h.c. . }
$$

As there are three up-type (charge $2 / 3$ ) quarks $u$ but $3+n_{N}$ down-type (charge $-1 / 3$ ) quarks $d, V$ is a $3 \times\left(3+n_{N}\right)$ rectangular matrix. It is of course not unitary, but it satisfies

$$
V V^{+}=1_{3} .
$$

$V$ is a sub-matrix of a $\left(3+n_{N}\right) \times\left(3+n_{N}\right)$ unitary matrix $[6,4]$

$$
\bar{V}=\left[\begin{array}{c}
V \\
B_{d}
\end{array}\right]
$$

where $B_{d}$ is a $n_{N} \times\left(3+n_{N}\right)$ matrix.

We introduce at this point a $3 \times 3$ sub-matrix $K$ of $V$. This is the $3 \times 3$ left-hand block of $V$, and it corresponds to the rnixing of the three up-type quarks with the known three down-type quarks, $d, s$ and $b$.

Defining

$$
\begin{gathered}
c_{L}^{d, w+1 !}=-1 / 2+1 / 3 \sin ^{2} \theta_{W}, \quad c_{L}^{\text {up }}=1 / 2-2 / 3 \sin ^{2} \theta_{W}, \\
c_{R}^{\text {down }}=1 / 3 \sin ^{2} \theta_{W}, \quad c_{R}^{\text {up }}=-2 / 3 \sin ^{2} \theta_{W},
\end{gathered}
$$

the neutral-current interactions are given by

$$
\mathcal{L}_{Z}=\frac{g}{\cos \theta_{W}} Z_{\mu}\left[-\frac{1}{2} \bar{d}_{L} \gamma^{\mu} \mathbf{D} d_{L}+G^{\mu}\right]
$$

Here,

$$
G^{\prime \prime}=c_{L}^{u p} \bar{u}_{L} \gamma^{\prime \prime} u_{L}+c_{R}^{u p} \bar{u}_{R} \gamma^{\prime \prime} u_{R}+\frac{1}{3} \sin ^{2} \theta_{\|} \cdot \bar{d}_{L} \gamma^{\prime \prime} d_{L}+c_{R}^{\text {doumn }} \bar{d}_{R} \gamma^{\prime \prime} d_{R}
$$

is a diagonal current, and

$$
\mathbf{D}=V^{\dagger} V
$$

is a hermitian $\left(3+n_{N}\right) \times\left(3+n_{N}\right)$ matrix.

The parameters

$$
\mathrm{D}_{i j}=\sum_{k=1}^{3} V_{k i}^{*} V_{k j}
$$

are denoted in the papers of $\mathrm{Nir}$ and Silverman $[4,5]$ by $U_{i j}$. These parameters, with $i \neq i$, govern the strength of the FCNC, given by the off-diagonal couplings of the $Z$ :

$$
-\frac{g}{2 \cos \theta_{11}} Z_{\mu}, \mathrm{D}_{, j} \bar{d}_{l, i} \boldsymbol{\gamma}^{\prime \prime} d_{l, j}
$$

for $i \neq j$. In practice, we will only be interested in the $3 \times 3$ sub-matrix of $D$ describing the FCNC of the known down-type quarks. Notice that the existence of these flavorchanging neutral couplings is directly related to the non-unitarity of the CKM matrix $V$, and that if one knows the charged-current couplings (the matrix $V$ ) then one also knows the neutral-current couplings (see Eq. 8). These are indeed features of a very general class of models. In the Appendix we explain how these features also apply in models with mirror quarks and vector-like isodoublet quarks. 


\section{Wolfenstein-type parametrization of $K$}

In general, there are various possible ways of parametrizing the CKM matrix. One may parametrize it by means of Euler angles and complex phase factors [7]; one may instead use as parameters the values of some rephasing-invariant quantities $[8,9]$; or one may use a Wolfenstein-type parametrization [10], in which some of the matrix elements themselves are used as parameters [11]. The latter type of parametrization always involves, because some unitarity conditions are quadratic in the matrix elements, matrix elements which are given as functions of the parameters as being the roots of some quadratic equations; in order to avoid that complication, a Wolfenstein-type parametrization is usually used in conjunction with an expansion in a small parameter $\lambda$, even though this expansion is not needed in principle. The expansion has also the advantage of allowing for a quick order-of-magnitude visualization of the various matrix elements.

In this section we develop a Wolfenstein-type parametrization of $K$. We are not interested in the whole matrix $V$, the dimension of which $\left[3 \times\left\{3+n_{N}\right)\right]$ we have not, even fixed, because we keep $n_{N}$ arbitrary. We are only interested in the left-side $3 \times 3$ sub-matrix of $V$. That is what we will parametrize. Furthermore, as we do not want to fix $n_{N}$, the unitarity of the extended $\left(3+n_{N}\right) \times\left(3+n_{N}\right)$ matrix $\bar{V}$ carnot help us; indeed, we can show that even for $n_{N}$ as low as three (which is the case in the $E_{0}$ model), the relevant $3 \times 3$ sub-matrix of $V, K$, has already the same number of parameters as a general $3 \times 3$ matrix. Therefore, the parametrization that we will present here does not include any unitarity assumption. Although it may look futile to be parametrizing a general matrix, this is not so in this case, first because we will be using a non-trivial expansion in a small parameter, and second because, as we shall show later, most of the parameters are experimentally tightly constrained.

Following Ref. [11], we first define the parameters $\lambda, A$ and $\mu$ from their relationships with the known moduli of the matrix elements $V_{u s}, V_{s b}$ and $V_{u b}$ :

$$
\begin{aligned}
& V_{12}=\lambda, \\
& V_{23}=A \lambda^{2}, \\
& V_{13}=A \mu \lambda^{3} e^{i \phi} .
\end{aligned}
$$

Experimentally [12], we know that $\lambda=0.2205 \pm 0.0018, A=0.88 \pm 0.16$ an $1 \mu=$ $0.45 \pm 0.14$. It is important that in our parametrization no parameter be much larger than 1 , because we intend to do a series expansion in $\lambda$, and large parameters would render that expansion meaningless. We choose a phase convention in which $V_{11}, V_{12}$, $V_{22}, V_{23}$ and $V_{33}$ are real and positive. Thus, the phase $\phi$ has a rephasing-invariant meaning: it is the phase of $\left(V_{13} V_{22} V_{12}^{*} V_{23}^{*}\right)$.

We now define three further real parameters, $B, C$ and $D$, by the following equations:

$$
\mathbf{D}_{11}=\sum_{k=1}^{3}\left|V_{k \cdot 1}\right|^{2}=1-B^{2} \lambda^{2}
$$




$$
\begin{aligned}
& \mathbf{D}_{22}=\sum_{k=1}^{3}\left|V_{k 2}\right|^{2}=1-C^{2} \lambda^{2}, \\
& \mathbf{D}_{33}=\sum_{k=1}^{3}\left|V_{k 3}\right|^{2}=1-D^{2} \lambda^{2} .
\end{aligned}
$$

These parameters express the lack of normalization of the columns of $K$. Because there is an extension of $K$ which is unitary of some dimension, we can guarantee that $B^{2}$, $C^{2}$ and $D^{2}$ are all positive. We will soon be determining the experimental bounds on these parameters; for the moment, it is enough to say that none of them may be much larger than unity.

Finally, we define three more parameters, $X, Y$ and $Z$, which are complex:

$$
\begin{aligned}
& \mathbf{D}_{21}=\sum_{k=1}^{3} V_{k 1} V_{k 2}^{*}=X \lambda^{4}, \\
& \mathbf{D}_{31}=\sum_{k=1}^{3} V_{k 1} V_{k \cdot 3}^{*}=Y \lambda^{4}, \\
& \mathbf{D}_{32}=\sum_{k=1}^{3} V_{k 2} V_{k \cdot 3}^{*}=Z \lambda^{4} .
\end{aligned}
$$

Once more, it is important to emphasize that the moduli of these three paramelers, which are directly related to the strength of the FCNC, will be found to be smaller than one.

We emphasize that the Eqs. 11 to 19 are all exact they are definitions of parameters. It is also clear that if we take $B=C=D=X=Y=Z=0$, we obtain the parametrization of Ref. [11] of the standard-mitel $3 \times 3$ CKM matrix.

The total number of parameters is 13: four phases ( $\phi$ and the phases of $X, Y$ and $Z$ ), and nine moduli ( $\lambda, A, \mu, B, C, D$, and the moduli of $X, Y$ and $Z$ ). This is equal to the maximum number of parameters for $K$, when that matrix is taken to be completely general - nine complex matrix elements, minus five phases which are rephased away. Indeed, we are just parametrizing a general matrix, without any unitarity constraints. The non-trivial thing about this job is the existence in that general matrix of some small quantities, some of which are small matrix elements, while others originate from the "almost-unitarity" of the matrix. On the other hand, it is evident that, if the number $n_{\Lambda}$ of exotic down-type quarks is sufficiently small, extra relations will exist among the parameters, due to the unitarity of the $\left(3+n_{N}\right) \times\left(3+n_{\wedge}\right)$ matrix $\bar{V}$, of which the observed $3 \times 3$ mixing matrix is a sub-matrix; an example of this will be presented in section 5 .

In genfral, for whatever number of exotic quarks (for whatever $n_{\wedge}$ ), we can only use the unitarity of $\hat{V}$ to derive some inequalities on the parameters of $K$. We use the fact that $K$ is a $3 \times 3$ sub-matrix of a larger (for definiteness, $m \times m$ ) unitary matrix $\tilde{V}$ to find that, for instance,

$$
B^{2} \lambda^{2}=\sum_{k=1}^{m}\left|\tilde{V}_{k 1}\right|^{2}
$$




$$
\begin{aligned}
C^{2} \lambda^{2} & =\sum_{k=4}^{m}\left|\bar{V}_{k 2}\right|^{2}, \\
X \lambda^{4} & =-\sum_{k=4}^{m} \tilde{V}_{k 1} \bar{V}_{k 2}^{*} .
\end{aligned}
$$

From these equations the following inequality is derived:

$$
|X|^{2} \lambda^{4} \leq B^{2} C^{2} \text {. }
$$

Similar inequalities of course exist for $|Y|$ and $|Z|$. If $|X|,|Y|,|Z|, B, C$ and $D$ are all of order 1 , then these inequalities are useless, because the powers of $\lambda$ guarantee that they are automatically satisfied. They might however become useful if it were found that $B, C$ or $D$ were extremely small. In particular, if two among the parameters $B$, $C$ and $D$ are zero there will be no FCNC.

It is possible to calculate exactly all the matrix elements of $K$ as functions of the parameters. $V_{33}$, which is real and pcsitive, is calculated from Eq. 16 (the normalization of the third column), and from Eqs. 12 and 13. In order to find $V_{22}$, one uses Eqs. 15, 16 and 19 (the orthogonality and the normalization of the second and third columns), to obtain [9]

$$
\begin{array}{r}
\left(\mathrm{D}_{33}-T_{13}\right) V_{22}^{2}+2 \operatorname{Re}\left(V_{12} V_{23} V_{13}^{*}-V_{23} \mathrm{D}_{32}\right) V_{22} \\
+\left|\mathrm{D}_{32}-V_{12} V_{13}^{*}\right|^{2}-\left(T_{12}-\mathrm{D}_{22}\right)\left(T_{13}+T_{23}-\mathrm{D}_{33}\right)=0,
\end{array}
$$

where $T_{i, j} \equiv\left|V_{i j}\right|^{2}$. This is a quadratic equation for $V_{22}$, which by definition is real and positive. One of the solutions of the equation is negative, and is therefore discarded; the other one gives the value of $V_{22}$. Having found $V_{22}$, we can calculate $V_{32}$ from Eq. 19. We now know all the matrix elements of the second and third columns of $K$. To calculate the matrix elements of the first column, we first use Eqs. 17 and 18 to write $V_{21}$ and $V_{31}$ as functions of $V_{11}$ and of known quantities. Then, substitution of the expressions for $V_{21}$ and $V_{31}$ into Eq. 14 yields a quadratic equation for $V_{11}$ :

$$
\begin{aligned}
& \left(\mathbf{D}_{22} \mathbf{D}_{33}-\left|\mathbf{D}_{32}\right|^{2}\right) V_{11}^{2} \\
& +2 \operatorname{Re}\left[\mathrm{D}_{21}\left(V_{13} \mathrm{D}_{32}-V_{12} \mathrm{D}_{333}\right)+\mathbf{D}_{31}\left(V_{12} \mathrm{D}_{32}^{*}-V_{13} \mathrm{D}_{22}\right)\right] V_{11} \\
& -\left|V_{22} V_{33}-V_{23} V_{32}\right|^{2} \mathrm{D}_{11}+\left|\mathrm{D}_{21}\right|^{2}\left(T_{23}+T_{33}\right)+\left|\mathrm{D}_{31}\right|^{2}\left(T_{22}+T_{32}\right) \\
& -2 \operatorname{Re}\left[\mathrm{D}_{21} \mathrm{D}_{31}^{*}\left(V_{22} V_{23}^{*}+V_{32} V_{33}^{*}\right)\right]=0 \text {. }
\end{aligned}
$$

This equations has a negative solution which we discard, since $V_{11}$ is by definition positive. The other solution gives $V_{11}$. The matrix elements $V_{21}$ and $V_{31}$ are then found from their expressions in terms of $V_{11}$.

This procedure enables us to calculate all the matrix elements of $K$ in terms of the parameters. The algorithm is exact but complicated, because of the presence of the roots of the quadratic equations for $V_{\mathrm{Jj}}, V_{22}$ and $V_{11}$. We simplify the result by . performing an expansion in powers of $\lambda$ to get

$$
V_{11}=1-\frac{1+B^{2}}{2} \lambda^{2}-\frac{\left(1-B^{2}\right)^{2}+4 C^{2}}{8} \lambda^{4}+\operatorname{Re} X \lambda^{5}
$$




$$
\begin{aligned}
& -\frac{\left(1-B^{2}\right)\left(1-B^{4}\right)+8 A^{2} \mu^{2}+4 C^{2}\left(1-B^{2}+2 C^{2}\right)}{16} \lambda^{6}+O\left(\lambda^{7}\right), \\
V_{22}= & 1-\frac{1+C^{2}}{2} \lambda^{2}-\frac{\left(1+C^{2}\right)^{2}+4 A^{2}}{8} \lambda^{4} \\
& -\frac{\left(1+C^{2}\right)^{3}-4 A^{2}\left(1+C^{2}-2 D^{2}\right)-16 A R e Z+16 A^{2} \mu \cos \phi}{16} \lambda^{6} \\
& +O\left(\lambda^{8}\right), \\
V_{33}= & 1-\frac{D^{2}}{2} \lambda^{2}-\frac{D^{4}+4 A^{2}}{8} \lambda^{1}-\frac{D^{6}+4 A^{2} D^{2}+8 A^{2} \mu^{2}}{16} \lambda^{6}+O\left(\lambda^{8}\right), \\
V_{21}= & -\lambda+\frac{B^{2}-C^{2}}{2} \lambda^{3}+X \lambda^{4}+\left(\frac{A^{2}}{2}+\frac{B^{4}}{8}+\frac{B^{2} C^{2}}{4}-\frac{3 C^{4}}{8}-A^{2} \mu e^{-i \phi}\right) \lambda^{5} \\
& +\left(A Y+\frac{C^{2} X-X^{*}}{2}\right) \lambda^{6}+O\left(\lambda^{\top}\right), \\
V_{: 31}= & A\left(1-\mu e^{-i \phi}\right) \lambda^{3}+Y \lambda^{4}+\frac{A}{2}\left[C^{2}+D^{2}-B^{2}+\mu e^{-i \phi}\left(1+B^{2}-D^{2}\right)\right] \lambda^{5} \\
& +\left(\frac{D^{2} Y}{2}-A X\right) \lambda^{6}+O\left(\lambda^{\top}\right), \\
& +O\left(\lambda^{8}\right) \cdot \\
& +\left[\frac{D^{2} Z}{2}-\frac{A D^{2} \mu}{2} e^{-i \phi}+\frac{A\left(1+2 C^{2}+C^{4}+2 D^{2}-3 D^{4}+2 C^{2} D^{2}\right)}{8}\right] \lambda^{6} \\
V_{32}= & -\left[\frac{A}{2}\left(1+C^{2}-D^{2}-2 \mu e^{-i \phi}\right)+Z\right] \lambda^{4} \\
= & -
\end{aligned}
$$

We emphasize that ours is a self-consistent approach: we first assume that $|X|,|Y|$, $|Z|, B, C$ and $D$ are all not larger than $\sim 1$, which assumption allows us to make an expansion in $\lambda$. In what follows, we will check that they are indeed experimentally constrained to be small. Not only does our parametrization reflect the power of the experimental bounds, as it is also suitable to adaptation, should these bounds improve in the future. For instance, if one would find that $1-\left|V_{11}\right|^{2}-\left|V_{21}\right|^{2}-\left|V_{31}\right|^{2}$ is $O\left(\lambda^{\prime}\right)$, one would just perform the substitution $B \rightarrow B \lambda$ in Eq. 14 and Eqs. 26 to 31, and the parametrizalion would be immediately updated.

\section{Experimental Constraints}

In this section we develop the various experimental constraints on the parameters introcluced in the last section.

\subsection{The normalization of the rows and columns of $V$}

The normalization of the first row of $V$ is dominated by the values [12] $\left|V_{11}\right|=0.9744 \pm$ 0.0010 and $\left|V_{12}\right|=0.2205 \pm 0.0018$. From these we get $1-\left|V_{11}\right|^{2}-\left|V_{12}\right|^{2}<0.0047$. 
Therefore,

$$
B^{2}+\left(C^{2}-B^{2}\right) \lambda^{2}+O\left(\lambda^{3}\right)<0.0975 .
$$

This equation provides the most stringent bound on $B$. Similarly, from $\left|V_{21}\right|>0.187$, $\left|V_{22}\right|>0.80$ and $\left|V_{23}\right|>0.036$, we obtain

$$
C^{2}+\left(B^{2}-C^{2}\right) \lambda^{2}+O\left(\lambda^{3}\right)<6.77
$$

We can get another limit on $B$ by considering the normalization of the first column of $V:$

$$
B^{2}<0.366 \text {. }
$$

From the normalization of the second column one obtains

$$
C^{2}<6.53
$$

It is clear that the bounds on $C^{2}$ of Eqs. 33 and 35 are unacceptably high (if $C^{2}$ were of order 6 , the series expansion in $\lambda$ performed in the last section would be meaningless). Fortunately, from the analysis of the decays of the $Z$ boson, it is possible to obtain a much better bound on $C^{2}$.

\subsection{The weak isospin of the bottom quark}

In a. recent study [13], Schaile and Zerwas have analysed the experimental constraints on the isospin values of the left-and right-handed $b$ quarks, $I_{3}^{L}(b)$ and $I_{3}^{R}(b)$ respectively, by combining the LEP data on the width $\Gamma[Z \rightarrow b \bar{b}]$ with the data for the forwardbackward assymetry of $b$ quarks found by LEP, PEP, PETRA and TRISTAN. They found

$$
\begin{aligned}
& I_{3}^{L}(b)=-.500_{-.002}^{+.0125} \\
& I_{3}^{R}(b)=.000_{-.028}^{+.028} .
\end{aligned}
$$

In our model there are no right-handed doublets, and therefore $I_{3}^{R}(b)=0$. On the other hand, using Eq. 6 , the bound on $I_{3}^{\prime}(b)$ corresponds to a bound on $\mathbf{D}_{33}=\sum_{i=1}^{3}\left|V_{i 3}\right|^{2}$. This bound is

$$
\sum_{i=1}^{3}\left|V_{i j}\right|^{2}>0.95
$$

Using our parametrization, this is

$$
D^{2}<1.05
$$

\subsection{The width of the $Z$}

In this model, the decay of the $\mathrm{Z}$ boson into hadrons has the width:

$$
\begin{aligned}
\Gamma[Z \rightarrow \text { hadrons }]_{U}= & 12 \Gamma[\nu \bar{\nu}]\left[\frac{1}{4} \sum_{i, j=1}^{3}\left|\mathbf{D}_{i j}-\frac{2}{3} \sin ^{2} \theta_{11} \cdot \delta_{i j}\right|^{2}\right. \\
& \left.+2\left(c_{l,}^{u p}\right)^{2}+2\left(c_{R}^{\text {up }}\right)^{2}+3\left(c_{R}^{\text {down }}\right)^{2}\right]
\end{aligned}
$$


where

$$
\Gamma[\nu \bar{\nu}]=\frac{G_{F} M_{Z}^{3}}{12 \sqrt{2} \pi}
$$

is the partial width of the $Z$ decaying into a neutrino pair, and the approximation of massless quarks is used. Notice that Eq. 40 includes as an essential assumption that exotic quarks are only present in the down-type-quark sector of the model. It is useful to introduce a parameter $\chi$, which measures the deviation of the width of the $Z$ from the $S M$ result:

$$
\begin{aligned}
\chi & \equiv \frac{1}{4} \sum_{i, j=1}^{J}\left|D_{i j}-\frac{2}{3} \sin ^{2} \theta_{1}, \delta_{i j}\right|^{2}-3\left(c_{I^{\prime}}^{\text {denun }}\right)^{2} \\
& =c_{l .}^{\text {doun }}\left(B^{2}+C^{2}+D^{2}\right) \lambda^{2}+\frac{B^{1}+C^{4}+D^{1}}{4} \lambda^{4}+\frac{|X|^{2}+|Y|^{2}+|Z|^{2}}{2} \lambda^{8} .
\end{aligned}
$$

Eq. 42 shows that $\chi=0$ in the SM, and Eq. 43 gives $\chi$ in our parametrization. The definition of $c_{l}^{\text {dimin }}$ was given in Eq. 4.

Before we compare with the experimental result, we must include the QCD corrections [14], which ammount to multiplicative factors of $1.040 \pm .007$ for all $Z$ decays, except for $\Gamma[Z \rightarrow b \bar{b}]$, for which the multiplicative factor is $1.045 \pm .007$. The difference in the QCD correction is due to the weak-vertex corrections involving a loop with the $W$ boson and the top quark. To get the total width of the $Z$, we simply add to $\Gamma_{h}$ the leptonic contributions, which we take to be the same as in the SM (i.e., we assume that there are no modifications of the leptonic sector of the SM). From the hadronic width we find

$$
-0.014<\chi<0.011
$$

while from the total width we have

$$
-0.012<\chi<0.013
$$

Neglecting the small contributions of the terms of order higher than $\lambda^{3}$ in Eq. 43 , this translates to

$$
B^{2}+C^{2}+D^{2}<0.69
$$

from the hadronic width. From the total width we derive the stronger bound

$$
B^{2}+C^{2}+D^{2}<0.59
$$

It is clear that the bounds on $C^{2}$ in Eqs. 46 and 47 are much stronger than the previous bounds in Eqs. 33 and 35. However, the stronger bound derived from the total width includes extra assumptions about the restricted fermion content of the model, namely, that there are no exotic leptons to which the $Z$ might decay. The hadronic width provides a slightly weaker bound Lut involves no such assumptions. 


\subsection{The Flavor-Changing Neutral Currents}

We now consider the contraints from the FCNC. These arise in processes involving the vertices in Eq. 10. Nir and Silverman [4] have done an extensive study of such processes, which was afterwards updated by Silverman [5] to include the new UA1 [15] bound on $B R\left(B \rightarrow \mu^{+} \mu^{-} X\right)$. In the present analysis we also include the recent [16] limit $B R\left[K^{+} \rightarrow \pi^{+} \nu \bar{\nu}\right]<5 \times 10^{-9}$, since it provides a bound on $\left|\mathrm{D}_{d_{s}}\right|$ that is better than the ones arising from $\epsilon$ and $\Delta M_{K}$. Furthermore, contrary to the analysis in $[4,5]$, we also take into account the standard-model contributions to the various processes for which they can reproduce the experimental results, with a suitable choice of parameters. There is an extensive literature [17] discussing the formulas for the SM contributions to the FCNC processes. Our aim is to obtain the most conservative limits possible. We have consistently taken the parameters, within reasonable ranges, to be such that one obtains the worst constraints consistent with experiment.

Particular attention has been paid to the decay $K_{L} \rightarrow \mu^{+} \mu^{-}$. This process is important because it gives a correlation between the magnitude and the phase of $\mathbf{D}_{d s}$. In models with down-type singlet quarks, we have the usual short-and long-distance SM contributions, and a new-physics contribution at tree level due to the FCNC. The new-physics term contributes only to the dispersive part of the amplitude and, since the decay is a two-(equal-)body-final-stale decay, and $K$ is a pseudoscalar, only the axial coupling survives. Therefore Eq. 3.6 of Reference [4] is incorrect by aproximately a factor of two. (This is however not the case for the three-body-final-state decays of $K$ or $B$ decaying to $\mu^{+} \mu^{-} X$.) As is well known, the absorptive part of the SM contributions to this decay is dominated by the two-photon intermediate state, which can be calculated in a model-independent way by relating it to the decay $K_{L} \rightarrow \gamma \gamma$. Subtracting this absorptive contribution from the experimentally observed width, we find that the dispersive contributions must add to $B R_{\text {disp }}=(0.5 \pm 0.7) \times 10^{-9}$. In the SM, this dispersive part has two origins: an electroweak short-distance term, and a long-distance term due to the two-photon intermediate state. The short-distance term grows with the masses of the top and charm quarks, and with $\operatorname{Re}\left(V_{t d}^{*} V_{l s}\right)$. Taking the extreme values of the relevant parameters to be $m_{t}=200 \mathrm{GeV}, m_{c}=1.7 \mathrm{GeV}$, $\lambda=0.2223, A=1.04$ and $\mu=0.59$, we find $B R\left(K_{L} \rightarrow \mu^{+} \mu^{-}\right)_{S D}<11.4 \times 10^{-9}$. The long-distance term has been the subject of much debate $[18,19,20,21]$. For instance, in reference $[19]$ it is found that the dispersive part of the two-photon amplitude may be as large as 'ialf of the absorptive part, and moreover, that the short-distance and longdistance dispersive amplitudes are likely to he of opposite signs. Since this question is not settled, we will admit [20] that the absorptive and dispersive contributions from the two-photon intermediate state may be of equal magnitude: $B R\left(K_{L} \rightarrow \mu^{+} \mu^{-}\right)_{L n}<$ $7.1 \times 10^{-9}$. We require the new-physics amplitude to be smaller than the sum of the amplitudes corresponding to the branching ratios $11.4 \times 10^{-9}, 7.1 \times 10^{-9}$ and $1.2 \times 10^{-9}$. We thus get.

$$
\left|\operatorname{ReD} D_{t s}\right|<3.1 \times 10^{-5} \text {. }
$$

For comparison we also consider two simplified cases. In case 1 we neglect altogether 


\begin{tabular}{|c|c|c|c|}
\hline System & Process & Quantity & Bound \\
\hline \multirow{5}{*}{ K mesons } & $\vec{K} \bar{K}$ mixing & $\Delta M_{K}$ & $\left|\operatorname{Re}\left(\mathrm{D}_{d s}\right)^{2}\right|<1.3 \times 10^{-6}$ \\
\hline & CP violation & $\epsilon$ & $\left|\operatorname{Im}\left(\mathbf{D}_{d_{s}}\right)^{2}\right|<1.7 \times 10^{-8}$ \\
\hline & \multirow[t]{2}{*}{$K \rightarrow \mu^{+} \mu^{-}$} & $\Gamma\left[K_{l}, \rightarrow \mu^{+} \mu^{-}\right]$ & $\left|\operatorname{ReD} D_{d s}\right|<3.1 \times 10^{-5}$ \\
\hline & & $\overline{\Gamma\left[K_{S} \rightarrow \mu^{+} \mu^{-}\right]}$ & $\left|\operatorname{ImD} D_{d s}\right|<1.9 \times 10^{-3}$ \\
\hline & $K^{+} \rightarrow \pi^{+} \nu \bar{\nu}$ & $\frac{\Gamma\left[k^{+}-\pi^{+} \nu 0 \mid\right.}{\Gamma \mid k^{+}-\pi^{2}+\varphi^{+}}$ & $\left|D_{d A}\right|<5.9 \times 10^{-5}$ \\
\hline \multirow[t]{2}{*}{ B mesons } & $B \bar{B}$ mixing & $x_{n}$ & $\left|\mathbf{D}_{d b}\right|<0.0046$ \\
\hline & $B \rightarrow \mu^{+} \mu^{-} X$ & $\frac{T\left(H-\mu^{+} \mu^{-}|X|\right.}{\mid[B-\mu \nu||}$ & $\left(\left|\mathbf{D}_{\mu b}\right|^{2}+\left|\mathbf{D}_{s b}\right|^{2}\right)^{1 / 2}<0.0024$ \\
\hline
\end{tabular}

Table 1: Experimental limits on FCNC in the down-type-quark sector

the long-distance contribution to the dispersive part of the amplitude, obtaining

$$
\left|\operatorname{Re} D_{d s}\right|<1.9 \times 10^{-5} \quad(\text { case } 1),
$$

and in case 2 we neglect the standard-model short-cistance contribution, and get

$$
\left|\operatorname{ReD} D_{d \vartheta}\right|<1.6 \times 10^{-5} \quad(\text { case } 2) .
$$

For the mass difference of the neutral kaons, we took the viewpoint that the standard-model long-distance contribution may be as large as the short-distance one. We also took into account the fact that the same strong-interaction matrix elements are present in the short-distance contribution (the box diagram) and in the new-physics contribution (the $Z$-exchange diagram). This also applies for the $\mathrm{CP}$-violating parameter $\epsilon$, and the $B-\bar{B}$ mixing parameter $x_{l}$. For this reason, the most conservative limits on the new physics are obtained when those matrix elements are as small as possible.

Our results are sumarized in Table 1 . Since the constraint from $\Gamma\left[K_{S} \rightarrow \mu^{+} \mu^{-}\right]$on $\left|\mathrm{ImD}_{\text {d.s }}\right|$ is weaker than that arising from the other processes combined, we will drop it from our analysis. The constraints on $\mathbf{D}_{d \text {, from }} \Delta M_{K}$ and from $\epsilon$ are also ineffective, since they are weaker than the new constraint from $K^{+} \rightarrow \pi^{+} \nu \bar{\nu}$. The constraint from $x_{1 \prime}$ is also made ineffective by the UA1 result on $B R\left(B \rightarrow \mu^{+} \mu^{-} X\right)$. We will however keep these ineffectixe bounds in our analysis, for comparison purposes. It is fortunate that the most stringent bounds that we now have, the ones from $B R\left(B \rightarrow \mu^{+} \mu^{-} X\right)$ and from $B R\left(K^{+} \rightarrow \pi^{+} \nu \bar{\nu}\right)$, originate in experimental limits which are still much above the SM expectations.

We translate the bounds in Table 1 to bounds on $X, Y$ and $Z$, by dividing them by the appropriate powers of $\lambda$. From the $B$-meson systems we obtain

$$
\begin{aligned}
|Y| & <2.0 \\
\sqrt{|Z|^{2}+|Y|^{2}} & <1.1 .
\end{aligned}
$$

From the kaon system we have

$$
|X|<2.6 \times 10^{-2}
$$




$$
\begin{aligned}
\left|\operatorname{Re} X^{2}\right| & <2.4 \times 10^{-1}, \\
\left|\operatorname{Im} X^{2}\right| & <3.3 \times 10^{-3}, \\
|\operatorname{Re} X| & <1.4 \times 10^{-2} .
\end{aligned}
$$

The results in Eqs. 49 and 50 give $|\operatorname{Re} X|<8.5 \times 10^{-3}$ (case 1), and $|\operatorname{Re} X|<7.1 \times 10^{-3}$ (case 2), respectively.

Defining $\zeta=\arg X$, we find that the Eqs. 53 to 56 define an area in the $|X|-\zeta$ plane, which we show in Figure 1a. In Figure $1 \mathrm{~b}$ we compare the allowed region that we have found with the curves corresponding to cases 1 and 2.

\subsection{Summary}

We now summarize the various bounds. We have found bounds on all parameters, except on the phases of $Y$ and $Z$. The phase of $X$ is also arbitrary, but there is a bound which connects it with the magnitude of $X$ (see figure 1a). We found that both $\left|Y^{-}\right|$and $|Z|$ may be of order 1 (see Eqs. 51 and 52), but all other parameters cannot be much larger than $\lambda:|X|<0.026, B^{2}<0.098, B^{2}+C^{2}+D^{2}<.59$. Indeed, $|X|$ is even bounded by $B R\left(K^{+} \rightarrow \pi^{+} \nu \bar{\nu}\right)$ to be no larger than $\lambda^{2}$, a bound approximately one order of magnitude stronger than the one arising from the simultaneous consideration of $\Delta M_{k}$ and $\epsilon$ (see figure 1a). The fact that no parameter is larger than 1 guarantees the validity of our approach of parametrizing the arbitrary mixing matrix by means of an expansion in $\lambda$. It is also interesting that the present bounds already allow us to say that the matrix elements of $K$ are essentialy the same as in the three-generation SM. We emphasize that such strong bounds on the mixing matrix are possible in an exotic-quark model, but not in a four- or more-generations model, because of the fact that vector-like quarks lead to FCNC with strength governed by the CKM matrix itself, and these FCNC are tightly constrained.

\section{The case of only one vector-like singlet}

In this chapter we examine the special case in which only one vector-like down-type iso-singlet is added to the SM.

\subsection{The unitarity constraints}

We remind the reader [4] that $\bar{V}$ is in this case an unitary $4 \times 4$ matrix, the upper $3 \times 4$ sub-matrix of which is the CKM matrix $V$. Because of the $4 \times 4$ unitarity of $\dot{V}$, there are extra constraints on the mixing in this particular case. While $K$ in section 3 is a general matrix, with no unitarity constraints on it, and is therefore parametrized. by 13 parameters, in the present case that matrix is a sub-matrix of the $4 \times 4$ unitary matrix $\bar{V}$, which may be parametrized by only 9 parameters.

Let us consider once more the general parametrization of section 3 . The parameters expressing the lack of normalization of the columns of the matrix $K$ (viz. $B, C$ and 
$D)$ are now related to the magnitudes of the matrix elements of the fourth row of $\bar{V}$. For instance, $B \lambda=\left|\bar{V}_{41}\right|$, with similar expressions for $C$ and $D$. Similarly, the FCNC parameters can now be written as products of elements of the fourth row of $\bar{V}$ :

$$
\begin{aligned}
& X \lambda^{4}=-\bar{V}_{41} \bar{V}_{12}^{*}, \\
& Y \lambda^{4}=-\tilde{V}_{41} \bar{V}_{13}^{*}, \\
& Z \lambda^{4}=-\bar{V}_{42} \bar{V}_{43}^{*} .
\end{aligned}
$$

From these equations one obtains the four constraints. The constraint

$$
X Y^{* *} Z \lambda^{6}=-B^{2} C^{2} D^{2}
$$

reduces the number of independent phases by one, by saying that $X Y^{\star} Z$ must be a real negative number. On the other hand,

$$
\begin{aligned}
& |X| \lambda^{2}=B C \\
& |Y| \lambda^{2}=B D \\
& |Z| \lambda^{2}=C D
\end{aligned}
$$

reduce the number of independent moduli by three. Notice that these equations just say that the inequalities like Eq. 23 become, in the case of just one exotic quark, equalities. As an important consequence of the Eqs. 61 through 63, we obtain that at least two of the parameters $B, C$ and $D$ must in this case be $\sim \lambda$, or smaller.

\subsection{Experimental constraints}

We briefly discuss the constraints imposed by experiment on the (reduced) parameter space. As we saw in section 4, normalization of the first row of the observed CKM matrix implies $B<0.31$. We also found that the width of the $Z$ boson leads to $B^{2}+C^{2}+D^{2}<0.59$.

But now, the analysis of FCNC can be reexpressed in terms of the same parameters, yielding

$$
\begin{aligned}
B C & <1.2 \times 10^{-3}, \\
D \sqrt{C^{2}+B^{2}} & <0.05 .
\end{aligned}
$$

These bounds define a parameter space in which the maximum allowed value of $B$ is determined by the normalization of the first row of $K$, and the maximum allowed values of $C$ and $D$ are determined by the width of the $Z$. The FCNC provide bounds on the products of any two of these parameters. As we have seen in Fig. 1, there is also a bound connecting the product $B C$ and the phase $\zeta$.

We wish to emphasize that the constraints from the FCNC, although they provide very strong bounds on the products $B C, B D$ and $C D$, leave the parameters $B, C$ and $D$ independently unconstrained. For example, the present experimential constraints are consistent with $D$ as large as .7 , provided that $B$ and $C$ are of order $\lambda^{2}$. Therefore, the bounds arising from the normalization of the rows and columns of $\bar{V}$, and from the decay of the $Z$ boson, cannot be neglected. 


\section{Conclusions}

If vector-like iso-singlet quarks exist, the generalized CKM matrix is not unitary. The lack of unitarity is connected with the appearance of flavor-changing neutral vertices. This fact allows us to set very stringent bounds on the extra parameters of the generalized CKM matrix, even if we do not make any assumptions about the number of iso-singlet quarks existent, and therefore about the dimension of the unitary matrix $\bar{V}$ in which the CKM matrix is embedded. A similar possibility does not arise in a model with more than three generations, in which the observed part of the CKM matrix is also a sub-matrix of a larger unitary matrix $V$, but no FCNC exist.

We have emphasized the value of the constraints following from the analysis of the width of the $Z$ boson, and from the isospin value of the left-handed bottom quark: they are essential to bound some parameters, which would otherwise be left un-constrained by the analysis of the FCNC alone. The overall bounds now existent allow a selfconsistent Wolfenstein-type expansion, in a small parameter $\lambda$, of the usual $3 \times 3$ part of the whole CKM matrix, even without making any unitarity assumptions. That expansion shows that, for whatever number of extra iso-singlet down-type quarks existent, the usual $3 \times 3$ part of the CKM matrix must be practically the same as it was in the three-generation standard model, with the attendant constraints of $3 \times 3$ unitarity. We find this fact remarkable. We have called the attention to the strongness of the recent bound insuing from the non-observance of the decay $K^{+} \rightarrow \pi^{+} \nu \bar{\nu}$ at the $5 \times 10^{-9}$ level.

\section{A The mixing matrices}

Besides down-type isosinglet vector-like quarks, there may be various reasons for considering theories with other types of exotic quarks, in particular mirror quarks, or up-type isosinglet vector-like quarks [22]. It is useful to illustrate the general structure of the mixing matrices in a theory with all those kinds of exotic quarks put together. We find that the existence of flavor-changing couplings of the $Z$ boson and of the Higgs boson are in all cases directly related with the non-unitarity of the CabibboKobayashi-Maskawa (CKM) matrix.

The $S U(2) \times U(1)$ model with mirror fermions, vector-like singlet and vector-like doublet quarks has, in a weak basis, the following quark content:

$$
q_{I .}=\left(\begin{array}{c}
r_{L} \\
n_{l}
\end{array}\right), P_{l}, N_{l,}, p_{R}, n_{R}, q_{R}=\left(\begin{array}{c}
P_{R} \\
N_{R}
\end{array}\right) \text {. }
$$

$p$ and $P$ are used for quarks with charge $2 / 3$ (up-type), and $n$ and $N$ are used for charge $-1 / 3$ (down-type) quarks, when in a weak basis. In the mass basis we use, respectively, the letters $u$ and $d$. The right-handed doublets $q_{R}$ contain the right-handed components of the mirror quarks and of the iso-doublet vector-like quarks. The left-handed singlets $P_{l}$. and $N_{L}$ are the left-handed components of the iso-singlet vector-like quarks and of the mirror quarks. All these should be understood as vectors in generation space, 
with arbitrary dimensions. We of course assume that the total number of left-handed quark fields is equal to the total number of right-handed quark fields, separately in each charge sector, lest there should be massless quarks.

The Yukawa interactions with the Higgs doublet, and the invariant mass terms, are given by

$$
\begin{aligned}
\mathcal{L}_{\Lambda \prime}= & -\bar{q}_{L} H \Gamma n_{R}-\bar{q}_{L}\left(i \sigma_{2} H^{*}\right) \Delta p_{R}-\bar{N}_{L} H^{\dagger} \Pi q_{R}-\bar{P}_{L}\left(-i H^{T} \sigma_{2}\right) \Sigma q_{R} \\
& -\bar{N}_{L} M_{n} n_{R}-\bar{P}_{L} M_{\nu} p_{R}-\bar{q}_{L} M_{q} q_{R}+\text { h.c. },
\end{aligned}
$$

where $\Gamma, \Delta, \Pi$ and $\Sigma$ are matrices of Yukawa couplings, in generation space. Writing the Higgs field as:

$$
H=\left(\begin{array}{c}
G^{+} \\
\left(v+\rho+i G^{v}\right) / \sqrt{2}
\end{array}\right)
$$

we obtain

$$
\begin{aligned}
\mathcal{L}_{\Lambda \prime}-\mathcal{L}_{\rho}-\mathcal{L}_{G}= & -\frac{v}{\sqrt{2}}\left(\bar{n}_{L} \Gamma n_{H}+\bar{N}_{L} \Pi N_{K}+\bar{p}_{L} \Delta p_{R}+\bar{P}_{L} \Sigma P_{K}\right) \\
& -\bar{N}_{L} M_{n} n_{l}-\bar{n}_{L} M_{q} N_{R}-\bar{P}_{L} M_{\nu} p_{R}-\bar{p}_{L} M_{q} P_{R}+\text { h.c. },
\end{aligned}
$$

where $\mathcal{L}_{\phi}$ is the portion of $\mathcal{L}_{\Lambda}$ involving the Higgs scalar $\rho$, and $\mathcal{L}_{G}$ is the portion of $\mathcal{L}_{\Lambda /}$ involving the Goldstone bosons $G^{+}, G^{-}$and $G^{\prime \prime}$.

We tranform to the mass basis by means of unitary transformations:

$$
\begin{aligned}
& \left(\begin{array}{l}
n_{\alpha} \\
N_{u}
\end{array}\right)=\left(\begin{array}{l}
A_{d \alpha} \\
B_{d u}
\end{array}\right) d_{\alpha}, \\
& \left(\begin{array}{c}
p_{\alpha u} \\
P_{\alpha}
\end{array}\right)=\left(\begin{array}{l}
A_{u u} \\
B_{u r}
\end{array}\right) u_{n},
\end{aligned}
$$

where $\alpha$ is $L$ or $R$. The unitarity of the transformation matrices is equivalent to

$$
\begin{gathered}
A_{k \alpha}^{\dagger} A_{k \alpha}+B_{k \alpha}^{\dagger} B_{k \alpha}=1, \\
A_{k \alpha} A_{k \alpha}^{\dagger}=1, \quad B_{k \alpha} B_{k \alpha}^{\dagger}=1, \quad A_{k \alpha} B_{k \alpha}^{\dagger}=0,
\end{gathered}
$$

where $k$ is $u$ or $d$. The diagonalization conditions for the down-and up-type-quark mass matrices are, respectively,

$$
\begin{aligned}
& \left(\begin{array}{ll}
A_{d l l}^{\dagger} & B_{d l,}^{\dagger}
\end{array}\right)\left(\begin{array}{cc}
\frac{v}{\sqrt{2}} \Gamma & M_{u} \\
M_{n} & \frac{v}{\sqrt{2}} \Pi
\end{array}\right)\left(\begin{array}{c}
A_{d R} \\
B_{d R}
\end{array}\right)=M_{d}, \\
& \left(\begin{array}{ll}
A_{u L}^{\dagger} & B_{u L}^{\dagger}
\end{array}\right)\left(\begin{array}{cc}
\frac{1}{\sqrt{2}} \Delta & M_{n} \\
M_{n} & \frac{r}{\sqrt{2}} \Sigma
\end{array}\right)\left(\begin{array}{c}
A_{u R} \\
B_{u R}
\end{array}\right)=M_{u},
\end{aligned}
$$

where $M_{1}$ and $M_{1}$ are diagonal, real and non-negative. Defining ( $\alpha=L$ or $R$ )

$$
\begin{aligned}
V_{L} & \equiv A_{u L}^{\dagger} A_{d L}, \\
V_{l} & \equiv B_{u n}^{\dagger} B_{d R}, \\
\mathbf{U}_{u} & \equiv V_{u r} V_{r}^{\dagger}, \\
\mathbf{D}_{u} & \equiv V_{u}^{\dagger} V_{u},
\end{aligned}
$$


one easily finds that

$$
\begin{aligned}
\mathcal{L}_{\Lambda I}= & -\bar{d}_{L} M_{d} d_{R}-\bar{u}_{L} M_{u} u_{R} \\
& +\frac{\sqrt{2} G^{+}}{v}\left[\bar{u}_{L}\left(M_{u} V_{R}-V_{L} M_{d}+V_{L} M_{d} \mathrm{D}_{R}-\mathrm{U}_{L} M_{u} V_{R}\right) d_{R}\right. \\
& \left.+\bar{u}_{R}\left(M_{u} V_{L}-V_{R} M_{d}+V_{R} M_{d} \mathrm{D}_{L}-\mathrm{U}_{R} M_{u} V_{L}\right) d_{L}\right] \\
& +i \frac{G^{\prime \prime}}{v}\left[\bar{d}_{L}\left(M_{d} \mathrm{D}_{R}-\mathrm{D}_{l} M_{d}\right) d_{R}+\bar{u}_{L}\left(\mathrm{U}_{L} M_{u}-M_{u} \mathrm{U}_{R}\right) u_{R}\right] \\
& -\frac{\rho}{v}\left[\bar{d}_{L}\left(\mathrm{D}_{L} M_{d}+M_{d} \mathrm{D}_{R}-2 \mathrm{D}_{L} M_{d} \mathrm{D}_{R}\right) d_{R}\right. \\
& \left.+\bar{u}_{L}\left(\mathrm{U}_{L} M_{u}+M_{u} \mathrm{U}_{R}-2 \mathrm{U}_{L} M_{u} \mathrm{U}_{R}\right) u_{R}\right]+h . c .
\end{aligned}
$$

This equation gives the couplings of the Higgs scalar and of the Goldstone bosons. Similarly, the interactions of the fermions with the $Z$ boson, which in the weak basis are

$$
\begin{aligned}
\mathcal{L}_{7}= & \frac{g}{\cos \theta_{11}} Z_{\mu}\left[c_{L}^{u p} \bar{p}_{L} \gamma^{\mu} p_{L}+c_{R}^{u p} \bar{P}_{L .} \gamma^{\mu} P_{L}+c_{R}^{u p} \bar{p}_{R} \gamma^{\mu} p_{R}+c_{L}^{u p} \bar{P}_{R} \gamma^{\mu} P_{R}\right. \\
& \left.+c_{L}^{\text {down }} \bar{n}_{L} \gamma^{\mu} n_{L}+c_{R}^{\text {down }} \bar{N}_{L} \gamma^{\mu} N_{L}+c_{R}^{\text {down }} \bar{n}_{R} \gamma^{\mu} n_{R}+c_{L}^{\text {down }} \bar{N}_{R} \gamma^{\mu} N_{R}\right]
\end{aligned}
$$

(see Eqs. 4 and 5), are easily transformed into the mass basis, becoming

$$
\begin{aligned}
\mathcal{L}_{Z}= & \frac{g}{\cos \theta_{\|}} Z_{\mu}\left[-\frac{2}{3} \sin ^{2} \theta_{\|} \cdot \bar{u} \gamma^{\mu} u+\frac{1}{3} \sin ^{2} \theta_{\|} \cdot \bar{d} \gamma^{\mu} d\right. \\
& \left.+\frac{1}{2}\left(\bar{u}_{L} U_{L} \gamma^{\mu} u_{L}+\bar{u}_{R} U_{R} \gamma^{\prime \prime} u_{R}-\bar{d}_{L} D_{L} \gamma^{\mu} d_{L}-\bar{d}_{R} D_{R} \gamma^{\prime \prime} d_{R}\right)\right] .
\end{aligned}
$$

$V_{l}$, and $V_{l l}$ are the (generalized) Cabibbo-Kobayashi-Maskawa (CKM) matrices. Indeed, the charged-current Lagrangian reads

$$
\mathcal{L}_{\mathbb{1}}=\frac{g}{\sqrt{2}}\left(\bar{u}_{L} \gamma^{\mu} V_{L} d_{L}+\bar{u}_{R} \gamma^{\mu} V_{R} d_{R}\right) W_{\mu}^{+}+\text {h.c. . }
$$

The above Eqs. 74, 75, 77 and 78 emphasize an important feature of the theory $[6,23]$. The couplings of the $Z$ and of the Higgs scalar with the quarks are related to the couplings of the $W$, given by the generalized CKM matrices. The lack of unitarity of those CKM matrices induces flavor-changing neutral currents in the couplings of the $Z$ (and also in the couplings of the neutral Goldstone boson, and of the Higgs scalar).

The CKM matrices for the left-and right-handed currents, $V_{I}$, and $V_{R}$, are not unitary, and they are in general not even square matrices. However, using Eqs. 71 and 72 , we easily find that

$$
\begin{aligned}
& \dot{V}_{L} \equiv\left[\begin{array}{cc}
V_{I L} & B_{u L}^{\dagger} \\
B_{d l} & 0
\end{array}\right], \\
& \bar{V}_{R} \equiv\left[\begin{array}{cc}
0 & A_{d R} \\
A_{u R}^{\dagger} & V_{R}
\end{array}\right]
\end{aligned}
$$


are unitary [23]. This fact is useful when one wants to develop parametrizations for $V_{L}$ and $V_{k}$ Notice that the existence in $\bar{V}_{L}$ and $\bar{V}_{R}$ of sub-matrices which are null matrices renders the use of the usual Murnaghan parametrizations (parametrizations by Euler angles and complex phases) for $\bar{V}_{L}$ and $\bar{V}_{R}$ extremely difficult. In [23] the use of rephasing-invariant parametrizations was advocated. In this paper we have used instead a Wolfenstein-type parametrization, which has no problems with vanishing sub-matrices, and which has the advantage of allowing for a series expansion which simplifies it in practice.

We thank L. Wolfenstein for guidance throughout this work, and for reading the manuscript. We thank L.-F. Li for various discussions. We are also indebted to G. C. Branco and P. Parada for giving us a copy of their paper [23] prior to publication. This work was supported by the United States Department of Energy, under the contract DE-FG02-91ER-40682. The work of J.P. S. was partially supported by the Portuguese JNICT, under grant BIC $N^{0} \mathrm{C} / 14 / 91$.

\section{References}

[1] P. Langacker and D. London, Phys. Rev. D 38, 886 (1988).

[2] A. S. Joshipura, Ahmedabad report PRL-TH-91-18 (1991), unpublished.

[3] E. Nardi, E. Roulet and D. Tommasini, Fermilab report FERMILAB PUB-92/127A (1992), unpublished.

[4] Y. Nir and D. Silverman, Phys. Rev. D 42, 1477 (1990).

[5] D. Silverman, Phys. Rev. D 45, 1800 (1992).

[6] G. C. Branco and L. Lavoura, Nucl. Phys. B 278, 738 (1986).

[7] F. D. Murnaghan, The unitary and rotation groups, Spartan Books, Washington (1962).

[8] J. D. Bjorken and I. Dunietz, Phys. Rev. D 36, 2109 (1987).

[9] G. C. Branco and L. Lavoura, Phys. Lett. B 208, 123 (1988).

[10] L. Wolfenstein, Phys. Rev. Lett. 51, 1945 (1983).

[11] G. C. Branco and L. Lavoụra, Phys. Rev. D 38, 2295 (1988).

[12] Particle Data Group, M. Aguilar-Benitez et al, Phys. Rev. D 45, (June 1992, Part 2).

[13] D. Schaile and P. M. Zerwas, Phys. Rev. D 45, 3262 (1992). 
[14] F. A. Berends, in Z Physics at LEP I, eds. G. Altarelli, R. Kleiss and C. Verzegnassi, vol. I, pg. 89.

[15] UA1 Collaboration, C. Albajar et al, Phys. Lett. B 256, 163 (1991).

[16] E787 Collaboration represented by M. S. Atiya, talk presented at the Meeting of the Division of Particles and Fields of the American Physical Society in Vancouver (1991), unpublished.

[17] A. J. Buras and M. K. Harlander, Technische Universität München Report TUMT31-25/92 (1992), to appear in the review volume on Heavy Flavours, eds. A. J. Buras and M. Lindner, World Scientific Publishing Company, Singapore;

$\mathrm{Y}$. Nir, in Procedings of the Theoretical Advanced Study Institute in Elementary Particle Physics, World Scientific Publishing Company, Singapore (1992);

L. Wolfenstein, in Proceedings of the $4^{\text {th }}$ International Symposium on Heavy Flavour Physics, eds. M. Davier and G. Wormser, Editions Frontières, Gif-sur Yvette (1992).

[18] G. R. Harris and J. L. Rosner, Phys. Rev. D 45, 946 (1992)

[19] P. Ko, Phys. Rev. D 45, 174 (1992)

[20] C. S. Kim, J. L. Rosner and C. P. Yuan, Phys. Rev. D 42, 96 (1990)

[21] R. E. Shrock and M. B. Voloshin, Phys. Lett. B 87, 375 (1979)

[22] B. Mukhopadhyaya and S. Nandi, Phys. Rev. Lett. 66, 285 (1991);

W. S. Hou, Paul-Scherrer-Institut Report PSI-PR-91-36 (1991), unpublished;

T. P. Cheng and L. F. Li, Phys. Rev. D 45, 1708 (1992).

[23] G. C. Branco, T. Morozumi, P. Parada and M. N. Rebelo, SLAC report SLACPUB-5799 (1992), unpublished.

\section{FIGURE CAPTIONS}

Figure 1a: The allowed region for the parameter $X(\zeta \equiv \arg X)$ is the region below all the curves. The horizontal solid line comes from the upper bound on $B R\left(K^{+} \rightarrow\right.$ $\left.\pi^{+} l^{\prime} \bar{\nu}\right)$. The bounds from $B R\left(K_{l} \rightarrow \mu^{+} \mu^{-}\right), \epsilon$ and $\Delta M_{K}$ are represented by the the dashed, dotted and the dot-dashed curves, respectively.

Figure 1b: The allowed region in the $|X|-\zeta$ plane is the area below the solid line. It corresponds to the best bounds obtained from Figure 1a, the solid and dashed lines of that Figure. The other two curves are the less conservative bounds obtained from $B R\left(K_{l} \rightarrow \mu^{+} \mu^{-}\right)$in cases 1 (dotted curve) and 2 (dashed curve). 


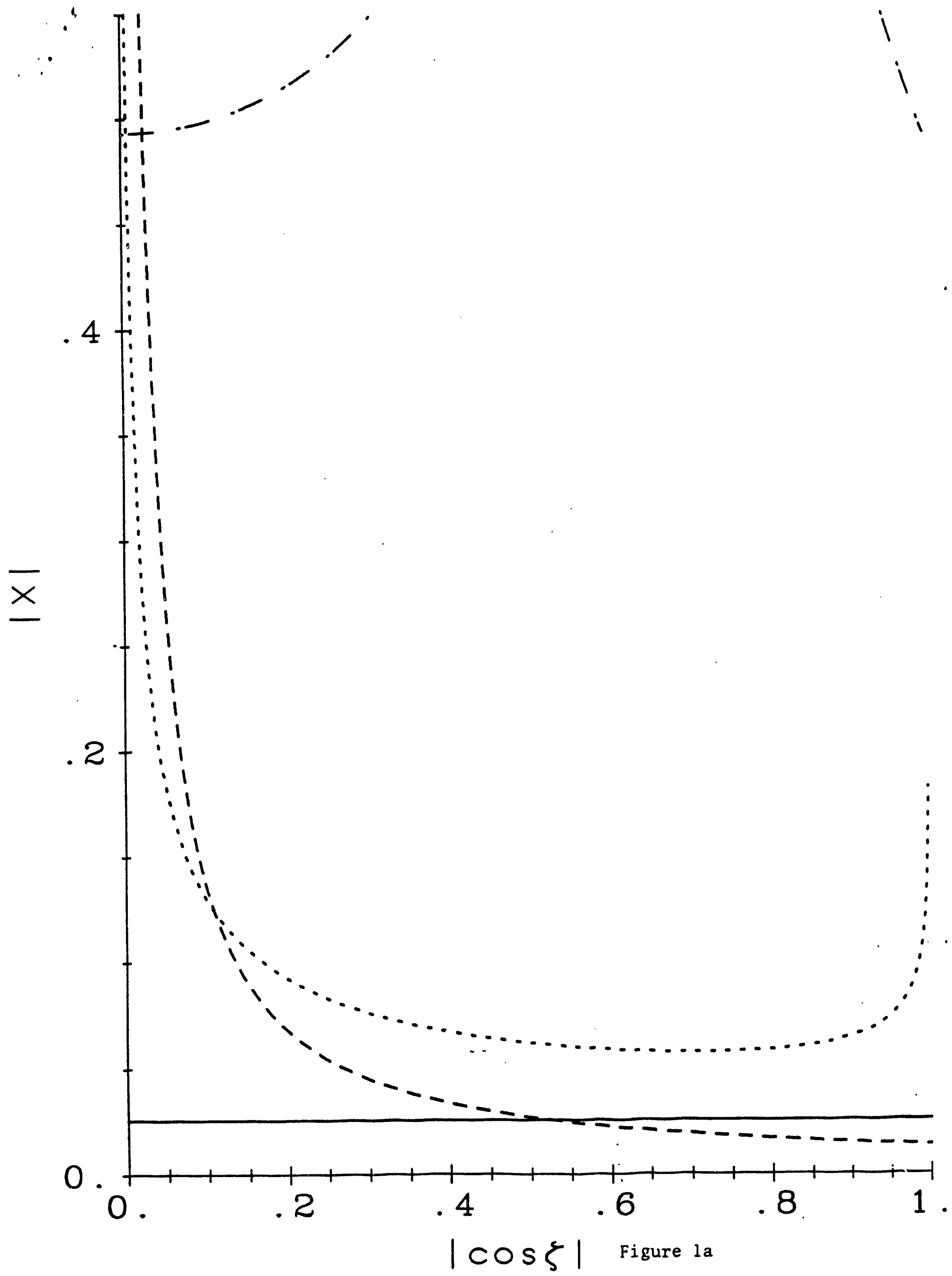




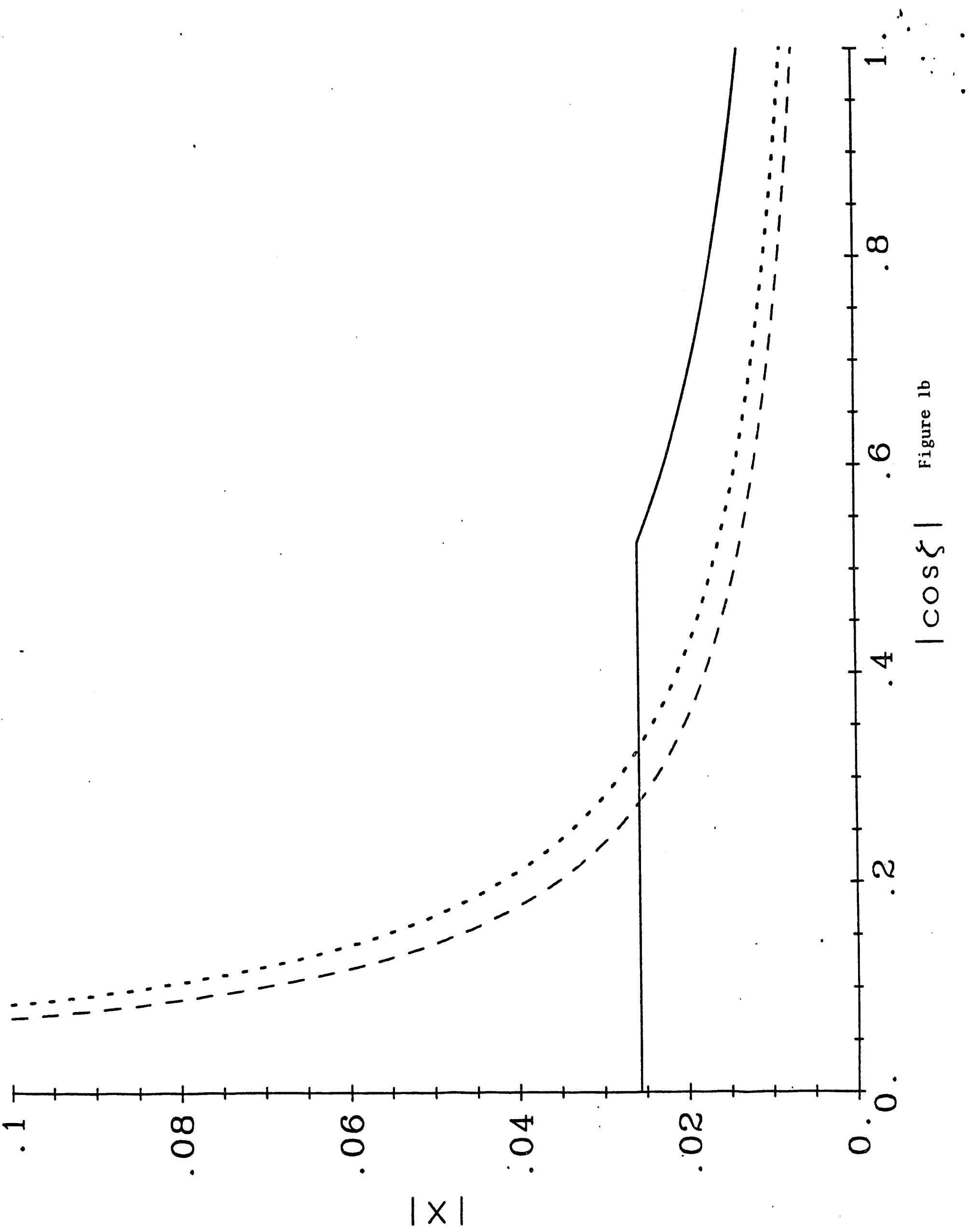


DATE FILMED $12117 / 92$ 
